\title{
Clinicopathological Characteristics of Ovarian Metastasis from Colorectal and Pancreatobiliary Carcinomas Mimicking Primary Ovarian Mucinous Tumor
}

\author{
CHEOL KEUN PARK ${ }^{1}$ and HYUN-SOO KIM ${ }^{2}$ \\ ${ }^{1}$ Department of Pathology, Armed Forces Capital Hospital, Seongnam, Republic of Korea; \\ ${ }^{2}$ Department of Pathology, Severance Hospital, Yonsei University College of Medicine, Seoul, Republic of Korea
}

\begin{abstract}
Background/Aim: A subset of carcinomas metastatic to the ovary can exhibit growth patterns closely resembling ovarian mucinous cystadenoma and borderline tumor. They can be misinterpreted as underlying primary ovarian mucinous tumors and can be erroneously used to suggest that the carcinomatous component arises from mucinous precursor lesions. Materials and Methods: We investigated the clinicopathological characteristics of 11 cases of metastatic carcinoma that had metastasized from colorectal and pancreatobiliary carcinoma and mimicked primary ovarian mucinous tumors. Results: The patient age ranged from 37 to 81 years old. Seven patients presented to gynecologists with nonspecific pelvic symptoms similar to those of primary ovarian tumors. The primary tumor was identified before the detection of the ovarian lesion in 6 cases, synchronously in 5, and postoperatively in 1 case. The ovarian tumors were bilateral in 6 cases. The greatest dimension of the metastatic tumors ranged from 4.8 to $23.0 \mathrm{~cm}$. Multinodularity was present in 7 cases, and surface involvement was identified in 5 cases. An infiltrative growth pattern was present, at least focally, in 8 cases. Six and 2 cases exhibited cystadenomatous and borderline-like growth patterns, respectively. Conclusion: Although the diagnosis of metastatic tumors to the ovary is possible in most cases on the basis of clinical presentation and standard diagnostic criteria, their differential diagnosis may be problematic because of morphological patterns that strikingly overlap with those of primary ovarian benign, borderline, and malignant mucinous tumors. The possibility of metastases should be considered when evaluating ovarian mucinous tumors.
\end{abstract}

Correspondence to: Hyun-Soo Kim, Department of Pathology, Severance Hospital, Yonsei University College of Medicine, 50-1, Yonsei-ro, Seodaemun-gu, Seoul 03722, Republic of Korea. Tel: +82 222281794, +82 23620860, e-mail: hyunsookim@yuhs.ac

Key Words: Ovary, mucinous tumor, metastasis, pancreaticobiliary, colorectal, adenocarcinoma.
The ovary is a common site of metastasis from a variety of primary tumors $(1,2)$. Although in most cases the primary tumors are diagnosed before the detection of the metastatic ovarian tumors, an ovarian mass can sometimes be the initial clinical manifestation of the primary disease. It is well established that primary epithelial tumors of the ovary can be mimicked by metastatic carcinoma from the abdominopelvic organs, leading to misdiagnosis (3). It is of utmost importance to correctly classify ovarian tumors as either primary or metastatic because the prognoses of these conditions differ significantly and determining the correct diagnosis has implications on the effective clinical management of patients $(2,4,5)$.

Among primary ovarian epithelial tumors, mucinous tumors pose the greatest difficulty with regard to distinction of primary from metastatic tumors (6). Metastatic carcinomas to the ovary are usually easily distinguished from primary ovarian mucinous tumors, including mucinous borderline tumors and carcinomas, when they exhibit characteristic gross and histological features. Primary ovarian mucinous tumors are typically large, unilateral, and multilocular cystic lesions, with smooth capsules. Moreover, primary ovarian mucinous carcinoma most often arises in association with mucinous cystadenoma and borderline tumors. Although primary mucinous ovarian carcinomas can exhibit destructive stromal invasion, they frequently show a dominantly expansile (confluent glandular) invasive pattern (7). In contrast, the typical features of metastatic carcinomas that distinguish them from primary ovarian mucinous tumors include a smaller size, bilaterality, involvement of the ovarian surface and superficial cortex, a multinodular growth pattern, and a dominantly infiltrative pattern of stromal invasion $(4,6,8,9)$.

A subset of metastatic carcinomas can manifest with one or more features suggesting a primary ovarian tumor both clinically and pathologically. These include i) presentation as a large, unilateral, multilocular cystic tumor lacking surface involvement, sometimes as the first manifestation of 
a disease with a clinically occult extraovarian source, and ii) certain growth patterns, including cystadenomatous, borderline-like, and expansile, which cannot be readily recognized as primary or metastatic. The metastases exhibiting borderline-like growth patterns often show obvious nuclear atypia and thus simulate ovarian mucinous borderline tumors with intraepithelial carcinoma. Metastases with small infiltrative foci simulate microinvasion derived from the adjacent mucinous borderline tumor. Furthermore, metastases with sufficiently crowded confluent glands, but lacking destructive stromal invasion simulate primary ovarian mucinous carcinomas with an expansile invasive pattern $(6,8,10-12)$. Despite recognition of the ability of these metastases to simulate primary tumors, and recent studies providing refined diagnostic criteria for primary ovarian mucinous tumors $(4,13,14)$, the problem of distinguishing these tumors remains unresolved. This issue is compounded by the fact that in routine practice, metastatic mucinous carcinomas are more common than primary ovarian mucinous carcinomas, with those of gastrointestinal and pancreaticobiliary origin being the most commonly encountered types $(15,16)$.

Herein, we comprehensively describe the clinicopathological characteristics of colorectal and pancreaticobiliary carcinomas that metastasized to the ovaries and mimicked primary ovarian tumors, with emphasis on the ability of these tumors to exhibit gross and histological features simulating primary ovarian tumors.

\section{Materials and Methods}

Case selection. Following approval (4-2018-0193) by the Institutional Review Board at the Severance Hospital, 11 cases of metastatic carcinoma to the ovary of colorectal (7 cases), pancreatic (3 cases), or extrahepatic biliary ( 1 case) origin were extracted from the surgical pathology files between July 2015 and December 2017. The diagnosis of an ovarian metastasis was based on standard diagnostic criteria $(4,9,13,17)$. The clinical features and intraoperative findings were obtained by review of the patients' electronic medical records or by communication with the referring gynecologists. Available preoperative and postoperative imaging materials were reviewed. In all cases, the primary tumor was available for histological examination. Pathology reports and gross photographs were also reviewed.

Pathological examination. The resected tissues were initially examined by pathologists before fixation in $10 \%$ neutral-buffered formalin. After fixation for 12-24 h, the tissues were examined macroscopically and sectioned. After processing with an automatic tissue processor (Peloris II, Leica Microsystems, Wetzlar, Germany), the sections were embedded in paraffin blocks. Four-micrometer-thick slices were cut from each formalin-fixed, paraffin-embedded tissue block and stained with hematoxylin and eosin using an automatic staining instrument (Ventana Symphony System, Ventana Medical Systems, Tucson, AZ, USA). After staining, the slides were covered with a glass coverslip and sent to 2 board-certified pathologists specialized in gynecological oncology for examination. They examined all available hematoxylin and eosin-stained slides using light microscopy (BX43 System Microscope, Olympus, Tokyo, Japan), made definite pathological diagnoses, and chose the most representative slide to perform immunohistochemical staining. For descriptive purposes, architectural growth patterns of the tumors were assessed as cystadenomatous, borderline-like, expansile, or infiltrative, on the basis of their resemblance to these patterns seen in primary ovarian mucinous tumors $(6,8,18)$. Nuclear atypia was assessed as mild, moderate, or severe. Additional pathological findings that were assessed included the presence of surface involvement, multinodular growth, extracellular mucin, and signet-ring cells.

Immunohistochemistry. The paraffin-embedded slices were deparaffinized and rehydrated with a xylene and alcohol solution. Immunohistochemical staining was performed using an automatic immunostaining instrument (Ventana Benchmark XT, Ventana Medical Systems) according to the manufacturer's recommendations (19-36). Antigen retrieval was performed using a Cell Conditioning Solution (CC1, Ventana Medical Systems). The slices were incubated with primary antibodies against cytokeratin 7 (CK7; 1:100; clone OV-TL 12/30; Dako, Glostrup, Denmark), CK20 (1:100; clone Ks20.8; Dako), and caudal-related homeobox 2 (CDX2; 1:400; clone EPR2764Y; Cell Marque, Rocklin, CA, USA). After chromogenic visualization using an ultraView Universal DAB Detection Kit (Ventana Medical Systems), slices were counterstained with hematoxylin. Appropriate positive and negative controls were concurrently stained to validate the staining method. Negative controls were prepared by substituting non-immune serum for the primary antibody and resulted in no detectable staining. For CK7 and CK20 immunostaining, staining with a moderate-to-strong intensity in the cell membrane was interpreted as positive expression. For CDX2 immunostaining, staining with a moderateto-strong intensity in the nuclei was interpreted as positive expression. Positive staining was considered diffuse when at least $50 \%$ of tumor cells were immunoreactive and focal when less than $50 \%$ of the cells were stained.

\section{Results}

Clinical features. The patients' ages ranged from 37 to 81 years (median, 51 years). There was no history of malignancy before the detection of colorectal, pancreatobiliary, or ovarian tumors in any patient. Seven patients presented to gynecologists with nonspecific pelvic symptoms, including palpable pelvic masses, and abdominal pain, discomfort, and/or distension. The primary tumors and ovarian metastases were simultaneously detected using preoperative imaging studies (computed tomography or magnetic resonance imaging) and/or exploratory laparotomy in 4 cases. In 7 cases, the tumors presented asynchronously, with the primary tumor diagnosis preceding the ovarian tumor diagnosis in 6 cases. The exact time interval between the diagnosis of the primary tumor and that of the ovarian tumor was known for 4 asynchronous cases. For the 4 cases with the primary tumor diagnosis established first, the mean time interval to diagnosis of the ovarian tumor was 19 months (range $=4-56$ months). In the remaining synchronously presented case, the primary 
tumor was diagnosed by follow-up investigations after ovarian resection. In this case, the clinical presentation was an ovarian mass, which was thought to be ovarian cancer upon clinical assessment. Pancreatic carcinoma was diagnosed 6 months after the ovarian tumor was removed. Extra-abdominal metastases sites included the lung in 2 cases and the vertebrae in 1 case.

Radiologically, the initial impressions of the ovarian tumors were primary ovarian cystic tumor in 6 cases, and 2 cases were diagnosed as primary ovarian cystic tumor versus metastasis. The remaining 3 cases were diagnosed as ovarian metastasis. The greatest dimension of tumors ranged from 4.8 to $23.0 \mathrm{~cm}$ (median, $11.4 \mathrm{~cm}$ ).

All patients except 1 underwent unilateral (3 patients) or bilateral (8 patients) salpingo-oophorectomy. Six of the 8 patients who underwent bilateral salpingo-oophorectomy underwent hysterectomy concurrently. Peritoneal carcinomatosis was observed in 5 patients and ascites in 7 patients. Intraoperative frozen sections of the ovarian masses were requested in 4 cases. An intraoperative diagnosis of metastatic adenocarcinoma was made in 2 cases, and 2 cases were diagnosed as at least mucinous borderline tumors. The initial histopathological diagnoses of the ovarian tumors were primary mucinous carcinoma in 2 cases and mucinous borderline tumor in 1 case. In the other cases, metastatic tumors were either diagnosed (7 cases) or favored (1 case).

Eight patients received postoperative chemotherapy. Two experienced progression of the disease and were lost to followup after 6 months. All the remaining patients experienced disease progression and died of their disease (8 patients); 1 of these patients was lost to follow-up after a short time.

Pathological features. Bilateral ovarian involvement was present in 6 cases. The appearance of the external surface was recorded for 9 tumors and was noted to be smooth in 2 tumors, irregular in 2 tumors, studded with tumor nodules in 3 tumors, and ruptured in 4 tumors, with adhesions or fibrinous exudate in 5 tumors and focal hemorrhage in 3 tumors. The sectioned surfaces were predominantly solid in 2 tumors, solid and cystic in 5 tumors, and multilocular cystic in 4 tumors. The cystic contents were recorded as mucoid in 6 tumors, serosanguinous in 3 tumors, and serous in 2 tumors. Grossly identifiable areas of necrosis were recorded in 8 tumors.

All the tumors, to varying degrees, had foci that were indistinguishable from, or closely simulated, primary ovarian mucinous tumors when viewed in isolation. However, all of them also had 1 or more morphological features characteristic of metastatic tumors. Multinodular growth was seen in 7 tumors, all of which demonstrated foci of uninvolved ovarian tissue between the tumor nodules. The nodules were often present in the superficial cortex. Ovarian surface implants were observed in 5 of 9 cases for which sections of the external surface were available. In 2 cases surface involvement could not be definitively determined because of the lack of a specific gross description of this aspect, and a lack of evidence of a definitive ovarian surface on available slides. The surface implants typically consisted of irregularly infiltrating glands within a desmoplastic, sometimes hyalinized, stroma.

Histologically, dilated and occasionally markedly cystic glands set in a variable amount of stroma represented the dominant morphological pattern in many tumors (Figure 1A). Infiltrating foci consisting of angulated tubular glands, irregular-shaped tumor cell nests, or singly scattered tumor cells were observed in 8 tumors (Figure 1B). The infiltrative growth pattern was accompanied by cystadenomatous and/or borderline-like patterns in 8 tumors (Figure 1C). In areas of cystadenomatous pattern, a single layer of mucinous epithelium exhibited minimal or no nuclear atypia. Nuclear stratification was not identified. A cystadenomatous growth pattern was the only pattern observed in 1 case. In contrast, tumors exhibiting extensive borderline-like growth patterns were characterized by multiple cystic lesions and glands of various sizes, intraluminal infoldings and papillary epithelial projections, and nuclear stratification, without definitive stromal invasion (Figure 1D). Tumors with marked atypia simulated mucinous borderline tumors with intraepithelial carcinoma (Figure 1E). Moreover, tumors with small infiltrative foci adjacent to the borderline-like areas simulated microinvasive mucinous carcinoma. The borderline-like pattern was exclusive in 2 tumors. Complex papillary structures with little intervening stroma, which are indicative of an expansile invasive pattern, were prominent in 2 tumors (Figure 1F). An admixture of the aforementioned morphological features was common and resulted in a particularly varied appearance, even between individual slides from the same patient.

The lining epithelium of the glands and cysts demonstrated variable mucin production. Mucin-producing epithelial cells were observed at least focally in all tumors. They were usually tall and columnar with abundant intracytoplasmic mucin (Figure 2A). Signet-ring cells comprised greater than $10 \%$ of the tumor cell population in 4 tumors, warranting a diagnosis of Krukenberg tumor. In other cases, sporadic signet-ring cells were identified. Three tumors contained colloid carcinoma-like areas with epithelium floating within mucin (Figure 2B). Areas resembling an ovarian mucinous borderline tumor, with or without intraepithelial carcinoma, were observed in all tumors. Bland-appearing, single-layered mucinous epithelium was seen in 4 tumors. Areas showing a non-mucinous epithelium were present in 3 tumors. They exhibited 3 main appearances: i) nonspecific cuboidal or columnar epithelium lining glands and cysts with a variable degree of nuclear atypia; ii) gland-forming adenocarcinoma with a cribriform architecture or focal cord-like infiltration mimicking high-grade endometrioid adenocarcinoma (Figure 2C); and iii) undifferentiated carcinoma. 
The nature of the stroma and its prominence varied greatly, both within individual tumors and among the ovarian tumors as a group. Fibromatous stroma was present in 5 tumors, desmoplastic stroma in 3 tumors (Figure 2D), and hypocellular to hyalinized stroma in 3 tumors (Figure 2E). Luteinized stromal cells were seen in 7 tumors. Although the stromal component was typically overshadowed by the glandular proliferation, in occasional cases the presence of superficially located benign glands and cysts within expanded fibrous stroma led to a remarkable mimicry of primary ovarian adenofibroma or cystadenofibroma. In tumors with a less prominent stromal component, the relatively benign-appearing cysts mimicked a mucinous cystadenoma with low power magnification.

Intraluminal necrotic debris was observed in 9 tumors, and 7 tumors demonstrated a garland-like pattern of cribriform epithelium around the area of necrosis (Figure 2F). Broad zones of tumor cell necrosis were seen in 4 tumors. Ovarian lymphatic invasion was unequivocally identified in only 3 tumors, although striking involvement of the paratubal lymphatics was noted in some other cases. Intracytoplasmic hyaline globules were present in rare cases.

Immunophenotypical features. The epithelial cells in all cases examined showed variable staining intensity according to the primary origin. All cases of metastatic colorectal carcinoma showed strong and diffuse immunoreactivities for CK20 (Figure 3A) and CDX2 (Figure 3B). None of them exhibited CK7 expression (Figure 3C). In all cases of metastatic pancreatobiliary carcinoma CK7 expression was strong and diffuse (Figure 3D). CK20 immunoreactivity was weak and patchy (Figure 3E), and CDX2 immunoreactivity was absent in all cases of metastatic pancreatobiliary carcinoma (Figure 3F).

\section{Discussion}

Metastatic tumors involving the ovary account for $5-30 \%$ of ovarian malignancies (37). They continue to be the source of numerous problems in differential diagnosis for the pathologists and have been a subject of much interest in the last few decades (38-40). Although overall information on ovarian metastasis from gastrointestinal and pancreatobiliary carcinomas is limited, the phenomenon is probably more frequent than the number of reported cases would indicate. Even with preoperative imaging studies, the primary lesions were not always diagnosed before surgery (41). The operative findings frequently showed abnormalities in and around the primary site that raised suspicion of disease of non-ovarian origin; however, in some cases, the primary tumor was not recognized or had previously been removed. In 1 case in this study, the primary tumor was not detected until 6 months postoperatively despite repeated preoperative and postoperative abnormal computed tomographic scans.
The relatively occult nature of pancreatobiliary carcinoma has previously been described (42).

The median diameter of metastatic tumors was $11.4 \mathrm{~cm}$, and bilateral ovarian involvement was observed in $6(54.5 \%)$ cases. Multinodularity was observed in $63.5 \%$, and cystic change was identified in $81.8 \%$ of the cases. Histologically, all cases showed 1 or more morphological features indicative of metastatic carcinoma. However, a 'maturation' phenomenon, i.e. the progression from a cystadenomatous pattern through a borderline-like pattern to a carcinomatous component, and variable degrees of nuclear atypia were both observed in several cases (6). When cystadenomatous and/or borderline-like growth patterns are admixed with a carcinomatous component, these patterns may be considered underlying mucinous precursor lesions, leading to the misdiagnosis of metastatic carcinoma as primary ovarian mucinous carcinoma $(1,43)$.

It is difficult to classify an ovarian mucinous tumor as a primary or metastatic lesion based solely on histological features. Thus, several studies have been performed to find meaningful clinical and radiological features for classifying ovarian mucinous tumors $(4,16)$. According to a previous study applying the algorithm based on tumor size and laterality, all cases of ovarian metastasis that originated from the pancreaticobiliary tract were classified correctly as metastatic lesions (16). In contrast, this algorithm was not useful in diagnosing metastatic colorectal carcinoma. Instead, microscopic surface implants and an infiltrative growth pattern were found to be useful clues for diagnosing metastatic colorectal carcinoma (4).

In addition to a cystadenomatous or borderline-like growth pattern, a transition from bland-looking or mildly atypical nuclei to moderately-to-severely atypical nuclei was identified in several cases. Although this maturation phenomenon is one of the characteristic morphological features of primary ovarian mucinous carcinoma, previous studies have reported that approximately one-third of metastatic mucinous carcinomas exhibit the maturation phenomenon (4). Furthermore, if well-differentiated glands and cysts are scattered in fibrous stroma, the lesion may mimic ovarian mucinous adenofibroma or mucinous cystadenoma, depending on the amount of fibrous stroma (38). During the intraoperative frozen section examination, the maturation phenomenon can lead to the misdiagnosis of a metastatic lesion as primary ovarian mucinous tumor because of the limited amount of specimen. Thus, a combination of medical history, clinical information, and histopathology is crucial to avoid misdiagnoses (38).

Some previous studies have assessed the diagnostic value of immunohistochemical staining for distinguishing primary ovarian mucinous tumor from metastatic mucinous carcinoma. The application of immunostaining for CK7 and CK20 does not appear to be useful $(44,45)$. Instead of using the CK7/CK20 immunoprofile, recent studies have reported 


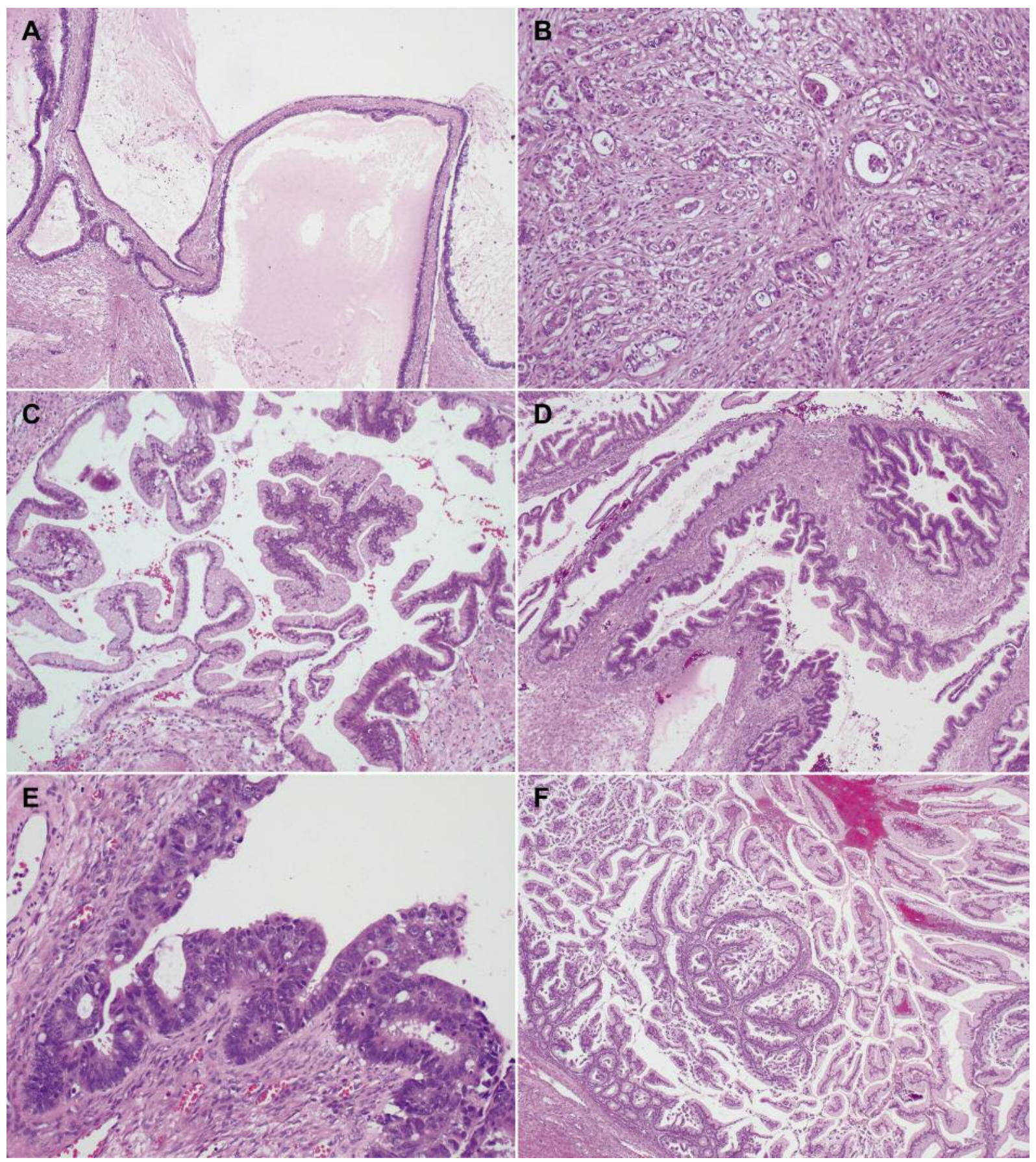

Figure 1. Histological features: growth pattern. A: Cystically dilated glands and intervening fibrous stroma. B: Variable-sized, irregular-shaped glands and individually scattered tumor cells. C: Areas of cystadenomatous and borderline-like patterns. D: Areas resembling ovarian mucinous borderline tumor. E: Areas resembling ovarian mucinous borderline tumor with intraepithelial carcinoma. F: Areas resembling ovarian mucinous carcinoma of expansile invasive pattern. Original magnification: A, x40;B, x100; C, x100; D, ×100; E, ×200; F, x40.

that overexpression of $\mathrm{CK} 17$ and reduction of deleted in pancreatic carcinoma 4 (DPC4) expression can be used as potential biomarkers for determining whether a lesion is of pancreaticobiliary origin $(46,47)$.
In conclusion, we investigated the clinicopathological features of 11 ovarian metastases from carcinomas of colorectal and pancreatobiliary origin. Although ovarian metastases can be diagnosed according to clinical features 

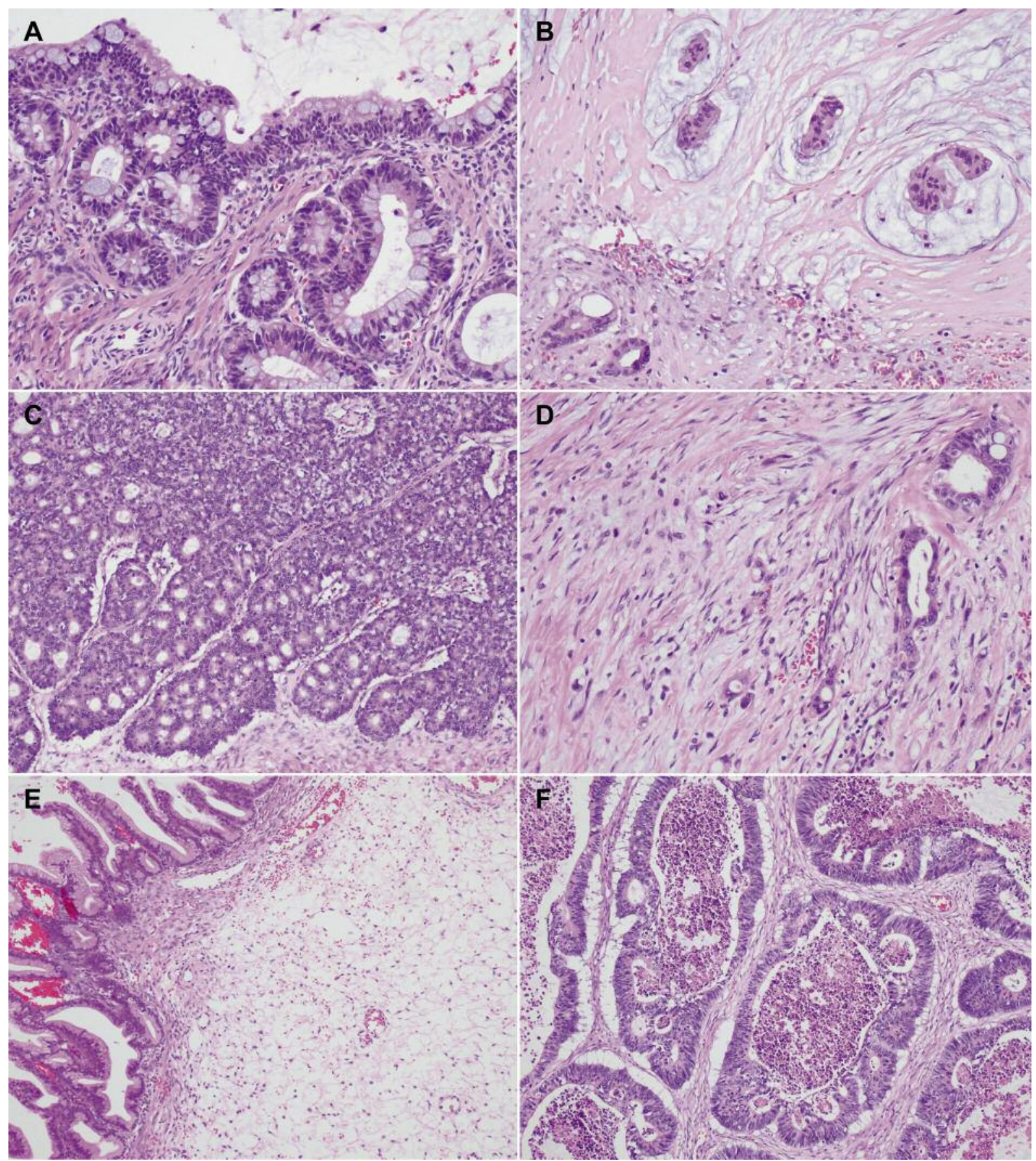

Figure 2. Histological features: lining epithelium and stroma. A: Tall columnar epithelium containing abundant intracytoplasmic mucin. B: Areas resembling colloid carcinoma. C: Areas resembling endometrioid carcinoma. D: Desmoplastic stromal reaction observed adjacent to infiltrating tumor glands. E: Hypocellular, edematous stroma. F: Large, cribriform glands with punctate central necrosis, resembling comedonecrosis of ductal carcinoma in situ of the breast. Original magnification: $A, \times 200 ; B, \times 100 ; C, \times 100 ; D, \times 200 ; E, \times 40 ; F, \times 100$.

and standard diagnostic criteria, their differential diagnosis can be challenging because of morphological patterns that overlap with those of primary ovarian mucinous tumors. Therefore, the possibility of metastatic lesions should be considered when evaluating ovarian mucinous tumors. In addition, further studies are necessary for developing an immunohistochemical panel to discriminate primary and metastatic mucinous tumors. 


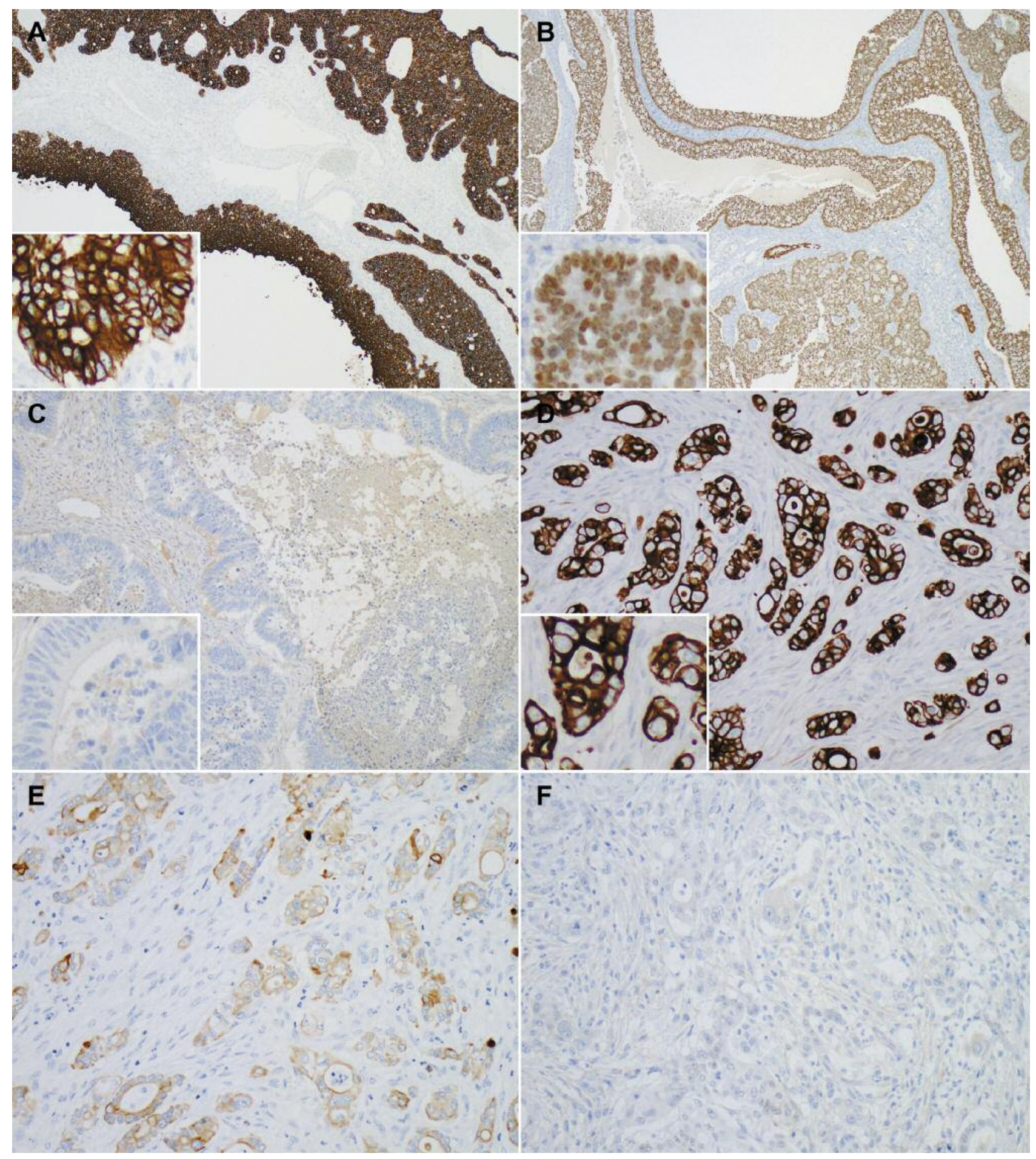

Figure 3. Immunohistochemical staining results. A and B: Strong and diffuse (A) cytokeratin 20 (CK20; membranous) and (B) caudal-related homeobox 2 (CDX2; nuclear) immunoreactivities in metastatic colorectal carcinoma. C: Lack of CK7 expression in metastatic colorectal carcinoma. D: Strong and diffuse membranous CK7 immunoreactivity in metastatic pancreatic carcinoma. E: Weak and patchy membranous CK20 expression in metastatic pancreatic carcinoma. $F$ : Lack of CDX2 expression in in metastatic extrahepatic cholangiocarcinoma. Original magnification: A, $\times 40 ; B, \times 40 ; C, \times 100 ; D, \times 200 ; E, \times 200 ; F, \times 200$. 


\section{Acknowledgements}

This research was supported by the Basic Science Research Program through the National Research Foundation of Korea (NRF) funded by the Ministry of Education (2016R1D1A1B03935584) and by a faculty research Grant of the Yonsei University College of Medicine (6-2018-0049).

\section{References}

1 Young RH and Hart WR: Metastases from carcinomas of the pancreas simulating primary mucinous tumors of the ovary. A report of seven cases. Am J Surg Pathol 13: 748-756, 1989.

2 Shiono S, Saito T, Fujii H, Arakawa A, Nakamura T and Yao T: A case of Krukenberg carcinoma metastasized from colon cancer resembling mucinous cystadenocarcinoma of the ovary. Int $\mathbf{J}$ Clin Exp Pathol 7: 394-401, 2014.

3 Khunamornpong S, Siriaunkgul S, Suprasert P, Pojchamarnwiputh $\mathrm{S}$, Na Chiangmai $\mathrm{W}$ and Young RH: Intrahepatic cholangiocarcinoma metastatic to the ovary: a report of 16 cases of an underemphasized form of secondary tumor in the ovary that may mimic primary neoplasia. Am J Surg Pathol 31: 1788-1799, 2007.

4 Lee KR and Young RH: The distinction between primary and metastatic mucinous carcinomas of the ovary: gross and histologic findings in 50 cases. Am J Surg Pathol 27: 281-292, 2003.

5 Lewis MR, Deavers MT, Silva EG and Malpica A: Ovarian involvement by metastatic colorectal adenocarcinoma: still a diagnostic challenge. Am J Surg Pathol 30: 177-184, 2006.

6 Meriden Z, Yemelyanova AV, Vang R and Ronnett BM: Ovarian metastases of pancreaticobiliary tract adenocarcinomas: analysis of 35 cases, with emphasis on the ability of metastases to simulate primary ovarian mucinous tumors. Am J Surg Pathol 35: 276-288, 2011.

7 Kurman RJ, Carcangiu ML, Herrington CS and Young RH: WHO Classification of Tumours of Female Reproductive Organs. Lyon, France: IARC, 2014.

8 Hart WR: Mucinous tumors of the ovary: a review. Int J Gynecol Pathol 24: 4-25, 2005.

9 Riopel MA, Ronnett BM and Kurman RJ: Evaluation of diagnostic criteria and behavior of ovarian intestinal-type mucinous tumors: atypical proliferative (borderline) tumors and intraepithelial, microinvasive, invasive, and metastatic carcinomas. Am J Surg Pathol 23: 617-635, 1999.

10 Elishaev E, Gilks CB, Miller D, Srodon M, Kurman RJ and Ronnett BM: Synchronous and metachronous endocervical and ovarian neoplasms: evidence supporting interpretation of the ovarian neoplasms as metastatic endocervical adenocarcinomas simulating primary ovarian surface epithelial neoplasms. Am J Surg Pathol 29: 281-294, 2005.

11 Ronnett BM, Yemelyanova AV, Vang R, Gilks CB, Miller D, Gravitt PE and Kurman RJ: Endocervical adenocarcinomas with ovarian metastases: analysis of 29 cases with emphasis on minimally invasive cervical tumors and the ability of the metastases to simulate primary ovarian neoplasms. Am J Surg Pathol 32: 1835-1853, 2008.

12 Judson K, McCormick C, Vang R, Yemelyanova AV, Wu LS, Bristow RE and Ronnett BM: Women with undiagnosed colorectal adenocarcinomas presenting with ovarian metastases: clinicopathologic features and comparison with women having known colorectal adenocarcinomas and ovarian involvement. Int J Gynecol Pathol 27: 182-190, 2008.

13 Lee KR and Scully RE: Mucinous tumors of the ovary: a clinicopathologic study of 196 borderline tumors (of intestinal type) and carcinomas, including an evaluation of 11 cases with 'pseudomyxoma peritonei'. Am J Surg Pathol 24: 1447-1464, 2000.

14 Rodriguez IM and Prat J: Mucinous tumors of the ovary: a clinicopathologic analysis of 75 borderline tumors (of intestinal type) and carcinomas. Am J Surg Pathol 26: 139-152, 2002.

15 Seidman JD, Kurman RJ and Ronnett BM: Primary and metastatic mucinous adenocarcinomas in the ovaries: incidence in routine practice with a new approach to improve intraoperative diagnosis. Am J Surg Pathol 27: 985-993, 2003.

16 Yemelyanova AV, Vang R, Judson K, Wu LS and Ronnett BM: Distinction of primary and metastatic mucinous tumors involving the ovary: analysis of size and laterality data by primary site with reevaluation of an algorithm for tumor classification. Am J Surg Pathol 32: 128-138, 2008.

17 Young RH and Scully RE: Differential diagnosis of ovarian tumors based primarily on their patterns and cell types. Semin Diagn Pathol 18: 161-235, 2001.

18 Ronnett BM, Kajdacsy-Balla A, Gilks CB, Merino MJ, Silva E, Werness BA and Young RH: Mucinous borderline ovarian tumors: points of general agreement and persistent controversies regarding nomenclature, diagnostic criteria, and behavior. Hum Pathol 35: 949-960, 2004.

19 Jang MI, Sung JY, Kim JY and Kim HS: Clinicopathological characteristics of metaplastic papillary tumor of the fallopian tube. Anticancer Res 37: 3693-3701, 2017.

$20 \mathrm{Na} \mathrm{K}$, Kim EK, Jang W and Kim HS: CTNNB1 mutations in ovarian microcystic stromal tumors: Identification of a novel deletion mutation and the use of pyrosequencing to identify reported point mutation. Anticancer Res 37: 3249-3258, 2017.

$21 \mathrm{Na}$ K, Park SY and Kim HS: Clinicopathological characteristics of primary ovarian adenomyoma: a single-institutional experience. Anticancer Res 37: 2565-2574, 2017.

22 Joo JW, Kim HS, Do SI and Sung JY: Expression of Zinc Finger and BTB domain-containing 7A in colorectal carcinoma. Anticancer Res 38: 2787-2792, 2018.

23 Jung YY, Sung JY, Kim JY and Kim HS: Down-regulation of BCell translocation gene 1 by promoter methylation in colorectal carcinoma. Anticancer Res 38: 691-697, 2018.

24 Sung JY, Jung YY and Kim HS: Clinicopathological Characteristics and KRAS mutation status of endometrial mucinous metaplasia and carcinoma. Anticancer Res 38: 2779-2786, 2018.

$25 \mathrm{Na} \mathrm{K}$ and Kim HS: Clinicopathological characteristics of fallopian tube metastases from primary endometrial, cervical, and nongynecological malignancies: a single institutional experience. Virchows Arch 471: 363-373, 2017.

$26 \mathrm{Na} \mathrm{K}$ and Kim HS: Clinicopathologic and molecular characteristics of mesonephric adenocarcinoma arising from the uterine body. Am J Surg Pathol, 2017. doi: 10.1097/PAS.0000000000000991. [Epub ahead of print]

$27 \mathrm{Na} \mathrm{K}$, Lee JY, Sung JY, Kim GM, Koo JS and Kim HS: Comparative clinicopathological and cytomorphological analyses of peritoneal carcinomatosis associated with metastatic breast carcinoma and primary peritoneal/ovarian carcinoma in patients with a history of breast carcinoma. Virchows Arch 2018. doi: 10.1007/s00428-018-2390-5. [Epub ahead of print] 
28 Chung T, Do SI, Na K, Kim G, Jeong YI, Kim YW and Kim HS: Stromal p16 overexpression in gastric-type mucinous carcinoma of the uterine cervix. Anticancer Res 38: 3551-3558, 2018.

$29 \mathrm{Na} \mathrm{K}$, Sung JY and Kim HS: Clinicopathological characteristics of high-grade squamous intraepithelial lesions involving condyloma acuminatum. Anticancer Res 38: 1767-1774, 2018.

$30 \mathrm{Na} \mathrm{K}$, Sung JY and Kim HS: TP53 mutation status of tuboovarian and peritoneal high-grade serous carcinoma with a wildtype p53 immunostaining pattern. Anticancer Res 37: 66976703, 2017.

31 Sung JY, Na K and Kim HS: Down-regulation of inositol polyphosphate 4-phosphatase Type II expression in colorectal carcinoma. Anticancer Res 37: 5525-5531, 2017.

32 Park CK and Kim HS: Clinicopathological characteristics of ovarian sclerosing stromal tumor with an emphasis on TFE3 overexpression. Anticancer Res 37: 5441-5447, 2017.

33 Do SI, Kim HS, Kim K, Lee H, Do IG, Kim DH, Chae SW and Sohn JH: Predictive value of sphingosine kinase 1 expression in papillary thyroid carcinoma. Anticancer Res 37: 5399-5405, 2017.

34 Kim JY, Na K and Kim HS: Clinicopathological characteristics of mitotically-active cellular fibroma of the ovary: a singleinstitutional experience. Anticancer Res 37: 2557-2564, 2017.

$35 \mathrm{Na} \mathrm{K}$, Sung JY and Kim HS: Stromal p16 overexpression in adult granulosa cell tumors of the ovary. Anticancer Res 37: 2437-2444, 2017.

36 Do SI, Yoon G, Kim HS, Kim K, Lee H, Do IG, Kim DH, Chae SW and Sohn JH: Increased brahma-related gene 1 expression predicts distant metastasis and shorter survival in patients with invasive ductal carcinoma of the breast. Anticancer Res 36 : 4873-4882, 2016.

37 Lobo J, Machado B, Vieira R and Bartosch C: The challenge of diagnosing a malignancy metastatic to the ovary: clinicopathological characteristics vary and morphology can be different from that of the corresponding primary tumor. Virchows Arch 470: 69-80, 2017.

38 Khunamornpong S, Lerwill MF, Siriaunkgul S, Suprasert P, Pojchamarnwiputh S, Chiangmai WN and Young RH: Carcinoma of extrahepatic bile ducts and gallbladder metastatic to the ovary: a report of 16 cases. Int J Gynecol Pathol 27: 366-379, 2008.
39 Young RH: From krukenberg to today: the ever present problems posed by metastatic tumors in the ovary: part I. Historical perspective, general principles, mucinous tumors including the krukenberg tumor. Adv Anat Pathol 13: 205-227, 2006.

40 Young RH: From Krukenberg to today: the ever present problems posed by metastatic tumors in the ovary. Part II. Adv Anat Pathol 14: 149-177, 2007.

41 Ayhan A, Guney I, Saygan-Karamursel B and Taskiran C: Ovarian metastasis of primary biliary and gallbladder carcinomas. Eur J Gynaecol Oncol 22: 377-378, 2001.

42 Garcia A, De la Torre J, Castellvi J, Gil A and Lopez M: Ovarian metastases caused by cholangiocarcinoma: a rare Krukenberg's tumour simulating a primary neoplasm of the ovary: a two-case study. Arch Gynecol Obstet 270: 281-284, 2004.

43 Young RH and Scully RE: Ovarian metastases from carcinoma of the gallbladder and extrahepatic bile ducts simulating primary tumors of the ovary. A report of six cases. Int J Gynecol Pathol 9: 60-72, 1990.

44 Rullier A, Le Bail B, Fawaz R, Blanc JF, Saric J and Bioulac-Sage P: Cytokeratin 7 and 20 expression in cholangiocarcinomas varies along the biliary tract but still differs from that in colorectal carcinoma metastasis. Am J Surg Pathol 24: 870-876, 2000.

$45 \mathrm{Ji} \mathrm{H}$, Isacson C, Seidman JD, Kurman RJ and Ronnett BM: Cytokeratins 7 and 20, Dpc4, and MUC5AC in the distinction of metastatic mucinous carcinomas in the ovary from primary ovarian mucinous tumors: Dpc4 assists in identifying metastatic pancreatic carcinomas. Int J Gynecol Pathol 21: 391-400, 2002.

46 Chu PG, Schwarz RE, Lau SK, Yen Y and Weiss LM: Immunohistochemical staining in the diagnosis of pancreatobiliary and ampulla of Vater adenocarcinoma: application of CDX2, CK17, MUC1, and MUC2. Am J Surg Pathol 29: 359-367, 2005.

47 Chuang SC, Lee KT, Tsai KB, Sheen PC, Nagai E, Mizumoto K and Tanaka M: Immunohistochemical study of DPC4 and p53 proteins in gallbladder and bile duct cancers. World J Surg 28 : 995-1000, 2004.
Received July 3, 2018

Revised July 16, 2018

Accepted July 17, 2018 